\title{
Practice-Based Competencies for Accreditation of and Training in Graduate Programs in Genetic Counseling
}

\author{
Beth A. Fine, ${ }^{1,5}$ Diane L. Baker, ${ }^{2}$ Morris B. Fiddler, ${ }^{3}$ \\ and ABGC Consensus Development Consortium ${ }^{4}$
}

In January 1996, the American Board of Genetic Counseling (ABGC) adopted 27 practice-based competencies as a standard for assessing the training of graduate students in genetic counseling. These competencies were identified and refined through a collective, narrative process that took place from January through November 1994, and included directors of graduate programs in genetic counseling, $A B G C$ board members and expert consultants. These competencies now form the basis of the document "Requirements for Graduate Programs in Genetic Counseling Seeking Accreditation by the American Board of Genetic Counseling" (American Board of Genetic Counseling, 1996). The competencies are organized into four domains and are presented and discussed in this article.

KEY WORDS: genetic counseling; practice-based competencies; graduate program accreditation.

\footnotetext{
${ }^{1}$ Department of Obstetrics and Gynecology, Northwestern University Medical School, Chicago, Illinois.

${ }^{2}$ Department of Human Genetics, University of Michigan, Ann Arbor, Michigan.

${ }^{3}$ School for New Learning, DePaul University, Chicago, Illinois.

${ }^{4}$ The Consortium includes Directors of established graduate programs in genetic counseling and members of the ABGC Board of Directors who participated in the Consensus Development Conference held in January, 1994: Diane Baker (University of Michigan/ABGC); Bonnie Baty (ABGC); Joan Burns (University of Wisconsin); Debra Collins (ABGC); Virginia Corson (ABGC); Beth Fine (Northwestern University/ABGC); Elizabeth Gettig (University of Pittsburgh); Verle Headings (Howard University); Jacqueline Hecht (University of Texas); Carl Huether (University of Cincinnati); Bonnie LeRoy (University of Minnesota); Joan Marks (Sarah Lawrence College); Anne Matthews (University of Colorado); Roberta Palmour (McGill University); Lorna Phelps (Medical College of Virginia); Kimberly Quaid (Indiana University); Joan Scott (ABGC); Ann Smith (ABGC); Helen Travers (ABGC); Judith Tsipis (Brandeis University); Ann Walker (University of California-Irvine/ABGC); Jon Weil (University of California-Berkeley); S. Robert Young (University of South Carolina); Randi Zinberg (Mount Sinai School of Medicine, New York).

${ }^{5}$ Correspondence should be directed to Beth A. Fine, Graduate Program in Genetic Counseling, Northwestern University Medical School, Ward Building 18-171 (W143), 303 East Chicago Avenue, Chicago, Illinois 60611.
} 


\section{INTRODUCTION}

An entry-level genetic counselor must demonstrate the practice-based competencies listed below to manage a genetic counseling case before, during, and after the clinic visit or session. Therefore, the didactic and clinical training components of a curriculum must support the development of competencies that are categorized into the following domains: Communication Skills; Critical Thinking Skills; Interpersonal, Counseling, and Psychosocial Assessment Skills; and Professional Ethics and Values. Some competencies may pertain to more than one domain. These domains represent practice areas that define activities of a genetic counselor. The facet below each competency elaborates on skills necessary for achievement of each competency. These elaborations should assist program faculty in curriculum planning, development, and in program and student evaluation.

\section{DOMAIN I: \\ COMMUNICATION SKILLS}

1. Can establish a mutually agreed upon genetic counseling agenda with the client.

The student is able to contract with a client or family throughout the relationship; explain the genetic counseling process; elicit expectations, perceptions, and knowledge; and establish rapport through verbal and nonverbal interaction.

2. Can elicit an appropriate and inclusive family history.

The student is able to construct a complete pedigree; demonstrate proficiency in the use of pedigree symbols, standard notation, and nomenclature; structure questioning for the individual case and probable diagnosis; use interviewing skills; facilitate recall for symptoms and pertinent history by pursuing a relevant path of inquiry; and in the course of this interaction, identify family dynamics, emotional responses, and other relevant information.

3. Can elicit pertinent medical information including pregnancy, developmental, and medical histories.

The student is able to apply knowledge of the inheritance patterns, etiology, clinical features, and natural history of a variety of genetic disorders, birth defects, and other conditions; obtain appropriate medical histories; identify essential medical records and secure releases of medical information.

4. Can elicit a social and psychosocial history.

The student is able to conduct a client or family interview that demonstrates an appreciation of family systems theory and dynamics. The student is able to listen effectively, identify potential strengths and weaknesses, and assess individual and family support systems and coping mechanisms. 
5. Can convey genetic, medical, and technical information including, but not limited to, diagnosis, etiology, natural history, prognosis, and treatment/management of genetic conditions, and/or birth defects to clients with a variety of educational, socioeconomic, and ethnocultural backgrounds.

The student is able to demonstrate knowledge of clinical genetics and relevant medical topics by effectively communicating this information in a given session.

6. Can explain the technical and medical aspects of diagnostic and screening methods and reproductive options including associated risks, benefits, and limitations.

The student is able to demonstrate knowledge of diagnostic and screening procedures and clearly communicate relevant information to clients. The student is able to facilitate the informed-consent process. The student is able to determine client comprehension and adjust counseling accordingly.

7. Can understand, listen, communicate, and manage a genetic counseling case in a culturally responsive manner.

The student can care for clients using cultural self-awareness and familiarity with a variety of ethnocultural issues, traditions, health beliefs, attitudes, lifestyles, and values.

8. Can document and present case information clearly and concisely, both orally and in writing, as appropriate to the audience.

The student can present succinct and precise case-summary information to colleagues and other professionals. The student is able to write at an appropriate level for clients and professionals and produce written documentation within a reasonable time frame. The student can demonstrate respect for privacy and confidentiality of medical information.

9. Can plan, organize, and conduct public and professional education programs on human genetics, patient care, and genetic counseling issues.

The student is able to identify educational needs and design programs for specific audiences, demonstrate public speaking skills, use visual aids, and identify and access supplemental educational materials.

\section{DOMAIN II: \\ CRITICAL-THINKING SKILLS}

1. Can assess and calculate genetic and teratogenic risks.

The student is able to calculate risks based on pedigree analysis and knowledge of inheritance patterns, genetic epidemiologic data, and quantitative genetics principles.

2. Can evaluate a social and psychosocial history.

The student demonstrates understanding of family and interpersonal dynamics and can recognize the impact of emotions on cognition and retention, as well as the need for intervention and referral. 
3. Can identify, synthesize, organize, and summarize pertinent medical and genetic information for use in genetic counseling.

The student is able to use a variety of sources of information including client/family member(s), laboratory results, medical records, medical and genetic literature and computerized databases. The student is able to analyze and interpret information that provides the basis for a differential diagnosis, a risk assessment, and the use of genetic testing. The student is able to apply knowledge of the natural history and characteristics/symptoms of common genetic conditions.

4. Can demonstrate successful case management skills.

The student is able to analyze and interpret medical, genetic, and family data; to design, conduct, and periodically assess the case management plan; arrange for testing; and follow up with the client, laboratory, and other professionals. The student should demonstrate understanding of legal and ethical issues related to privacy and confidentiality in communications about clients.

5. Can assess client understanding and response to information and its implications to modify a counseling session as needed.

The student is able to respond to verbal and nonverbal cues and to structure and modify information presented to maximize comprehension by clients.

6. Can identify and access local, regional, and national resources and services.

The student is familiar with local, regional, and national support groups and other resources, and can access and make referrals to other professionals and agencies.

7. Can identify and access information resources pertinent to clinical genetics and counseling.

The student is able to demonstrate familiarity with the genetic, medical, and social-science literature, and on-line databases. The student is able to review the literature and synthesize the information in a critical and meaningful way.

\section{DOMAIN III: INTERPERSONAL, COUNSELING, AND PSYCHOSOCIAL ASSESSMENT SKILLS}

1. Can establish rapport, identify major concerns, and respond to emerging issues of a client or family.

The student is able to display empathic listening and interviewing skills, and address clients' concerns.

2. Can elicit and interpret individual and family experiences, behaviors, emotions, perceptions, and attitudes that clarify beliefs and values.

The student is able to assess and interpret verbal and nonverbal cues and use this information in the genetic counseling session. The student is able to engage clients in an exploration of their responses to risks and options. 
3. Can use a range of interviewing techniques.

The student is able to identify and select from a variety of communication approaches throughout a counseling session.

4. Can provide short-term, client-centered counseling, and psychological support.

The student is able to assess clients' psychosocial needs and recognize psychopathology. The student can demonstrate knowledge of psychological defenses, family dynamics, family theory, crisis-intervention techniques, coping models, the grief process, and reactions to illness. The student can use open-ended questions; listen empathically; employ crisis-intervention skills; and provide anticipatory guidance.

5. Can promote client decision-making in an unbiased, noncoercive manner.

The student understands the philosophy of nondirectiveness and is able to recognize his or her values and biases as they relate to genetic counseling issues. The student is able to recognize and respond to dynamics, such as countertransference, that may affect the counseling interaction.

6. Can establish and maintain inter- and intradisciplinary professional relationships to function as part of a health-care delivery team.

The student behaves professionally and understands the roles of other professionals with whom he or she interacts.

\section{DOMAIN IV: \\ PROFESSIONAL ETHICS AND VALUES}

1. Can act in accordance with the ethical, legal, and philosophical principles and values of the profession.

The student is able to recognize and respond to ethical and moral dilemmas arising in practice and seek assistance from experts in these areas. The student is able to identify factors that promote or hinder client autonomy. The student demonstrates an appreciation of the issues surrounding privacy, informed consent, confidentiality, real or potential discrimination, and other ethical/legal matters related to the exchange of genetic information.

2. Can serve as an advocate for clients.

The student can understand clients' needs and perceptions and represent their interests in accessing services and responses from the medical and social service systems.

3. Can introduce research options and issues to clients and families.

The student is able to critique and evaluate the risks, benefits, and limitations of client participation in research; access information on new research studies; present this information clearly and completely to clients; and promote an informed-consent process. 
4. Can recognize his or her own limitations in knowledge and capabilities regarding medical, psychosocial, and ethnocultural issues and seek consultation or refer clients when needed.

The student demonstrates the ability to self-assess and to be self-critical. The student demonstrates the ability to respond to performance critique and integrates supervision feedback into his or her subsequent performance. The student is able to identify and obtain appropriate consultative assistance for self and clients.

5. Can demonstrate initiative for continued professional growth.

The student displays a knowledge of current standards of practice and shows independent knowledge-seeking behavior and lifelong learning.

\section{DISCUSSION}

The 27 competencies expected of entry-level genetic counselors have been adopted by the ABGC as the centerpiece of its requirements for the accreditation of graduate programs in genetic counseling. They emerged from the collective effort of program directors and members of the ABGC Board of Directors (for a more detailed discussion of the case-based narrative process, see Fiddler and Alicea, 1996; Fiddler et al., 1996). These practice-based competencies profile the contemporary genetic counselor and were derived through a process that promoted reflection on practice and current standards for specific cases. At the same time, the competencies are generalized to allow for interpretation in multiple and varied contexts today and into the foreseeable future.

The extent to which the competencies anticipate the professional skills and knowledge required for the future, nevertheless, is unpredictable. If the changes witnessed by the profession over the past decade continue on a similar or even steeper trajectory, it will be essential to periodically review the competencies for their effectiveness and appropriateness as a template for professional training and practice standards (Rosenthal, 1991). The process described in the accompanying article should serve as a framework for future revisions (Fiddler et al., 1996).

In the immediate future, the competencies will serve as criteria for program accreditation, i.e., programs will be accredited based on their capacity to demonstrate learning and assessment opportunities which will support the development of these competencies for each student. The ABGC has moved from the content-driven accreditation process established by the $A B M G$ which required lists of course content and clinical contact hours to an accreditation model that fosters the development of practice-based skills that integrate knowledge from several disciplines. 
There will be a variety of other uses for the competencies in addition to their service as criteria for program accreditation. Probably the most far-reaching will be their use as primary guides for curriculum design and revision. The use of the competencies for student performance assessments and practitioner evaluations will foster the collection of outcome measures for effective teaching, clinical supervision, and genetic counseling practice. Other functions will include stimulating research, guiding continuing education and professional development, and defining and redefining the landscape of genetic counseling to a variety of audiences. The broad use of outcome measures in accreditation has been suggested for allied health fields. Barr questions whether accreditation should, in fact, be used as a stimulus for educational reform and suggests that multidimensional outcome measures would promote increased autonomy and improved patient care (Barr, 1994). This first iteration of practice-based competencies will serve as a foundation for these and other considerations.

The most immediate implication of the adoption of practice-based competencies for program accreditation, however, lies in the demands they place on those responsible for curriculum design and delivery. By defining the outcomes of graduate education, the central responsibility of programs and their faculty to recognize and organize activities that support the development of each competency is highlighted. Education is not defined by courses alone, but by the integration of knowledge into behaviors practiced and shaped through clinical experiences, laboratory and field activities, classroom learning, and supplementary clinical activities such as case conferences, journal clubs, and rounds. Integrating these various sources of learning elevates the importance of assessment and the description of criteria for evaluating the demonstration of proficiency at a variety of levels for each competency. By setting forth the outcomes expected from professional training, students are encouraged to assume greater responsibility for their own professional development and self-assessment. Each of these topics has its own complexities and relative importance with which program faculty and staff will grapple as they move through the accreditation process and as they re-evaluate their curricula.

Genetic counseling can be considered one of the allied health professions, although it was not included in the recent study conducted by the Pew Health Professions Commission. However, the recommendations of the Commission reflect the impact of changes in health care on practice, education, and accreditation so that health care providers, including genetic counselors, can better meet the public's future health care needs. The Commission identified 17 broad "Practitioner Competencies for 2005" designed 
for all health professionals. Nine of these are directly addressed in the ABGC competencies; these include the ability to provide contemporary clinical care; participate in coordinated care; involve patients and families in the decision-making process; assess and use technology appropriately; manage information; provide counseling on ethical issues; participate in a racially and culturally diverse society; and continue to learn. Recommended strategies for allied health accreditation include encouraging continuous validation of practice and reassessing competency requirements. A continuous evaluation of consumer needs should drive accreditation by developing outcome measures that will ensure quality practice and protect the public (Finocchio, 1994).

The opportunity to learn from the experience of other allied health professions has greatly enhanced the $A B G C$ 's efforts in the area of accreditation. The development of practice-based competencies for genetic counseling is a first step toward identifying and meeting practice standards as we enter the twenty-first century.

The success of the competencies in guiding accreditation and training will ultimately be measured by the knowledge, skills, and attitudes demonstrated by new members entering the genetic counseling profession and the enthusiasm they generate within the field to interpret and support their development. We believe that these practice-based competencies were developed through a process that affirmed the clinical and pedagogical experience and voices of practitioners and educators. They represent a cross-section of the genetic counseling profession whose responsibilities for defining future directions for genetic counseling are accented by the new governance structure of the $\mathrm{ABGC}$.

\section{ACKNOWLEDGMENTS}

The authors gratefully acknowledge the American Board of Genetic Counseling, Inc. for supporting the Consensus Development Conference. We also thank Sharon Robinson and Randy Gresham for his assistance with the manuscript.

\section{REFERENCES}

American Board of Genetic Counseling (1996) Requirements for Graduate Programs in Genetic Counseling Seeking Accreditation by the American Board of Genetic Counseling, Bethesda, MD: American Board of Genetic Counseling.

Barr JT (1994) Patient-centered outcome measures of accreditation. $J$ Allied Health 23(1):47-49.

Fiddler MB, Alicea M (1996) Use of a collective narrative process to articulate practice-based advising competencies. $N A C A D A J$, in press. 
Fiddler MB, Fine BA, Baker DL, ABGC Consensus Development Consortium (1996) A Case-Based Approach to the Development of Practice-Based Competencies for Accreditation of and Training in Graduate Programs in Genetic Counseling. $J$ Genet Counsel 5(3):105-112.

Finocchio LJ (1994) Looking into the future of allied health: Recommendations of the Pew Health Professions Commissions. J Allied Health 23(1):29-34.

Rosenthal JP (ed) (1991) Committee on Allied Health Education and Accreditation (CAHEA) Accreditation Manual. Chicago: American Medical Association, pp 16-17. 